\title{
Erratum: Contextuality and noncontextuality measures and generalized Bell inequalities for cyclic systems [Phys. Rev. A 101, 042119 (2020)]
}

\author{
Ehtibar N. Dzhafarov (i), Janne V. Kujala, and Víctor H. Cervantes
}

(Received 21 April 2021; published 17 May 2021)

DOI: 10.1103/PhysRevA.103.059901

The measures $\mathrm{CNT}_{2}$ and $\mathrm{NCNT}_{2}$ are defined in the paper as the $L_{1}$ distance between a point representing a system of dichotomous random variables and the surface of the noncontextuality polytope. The values of the dichotomous variables can be chosen arbitrarily: Switching from the $\{0,1\}$ values to $\{a, b\}$ amounts to multiplying all values of $\mathrm{CNT}_{2}$ and $\mathrm{NCNT}_{2}$ by $(a-b)^{2}$. In our paper, $\mathrm{CNT}_{2}$ and $\mathrm{NCNT}_{2}$ were defined for $\{0,1\}$ variables, but when it was more convenient, we also used the $\{-1,+1\}$ labeling so that the $L_{1}$ distance in question was 4 times the value of $\mathrm{CNT}_{2}$ or $\mathrm{NCNT}_{2}$. By an oversight, in Secs. VIII and IX, where the $\{-1,+1\}$ labeling was used, the coefficient 4 was omitted. So every occurrence of $\mathrm{CNT}_{2}$ and $\mathrm{NCNT}_{2}$ in these sections should be multiplied by 4 to be consistent with their definition in earlier sections. This applies only to the text of these sections; the numerical values in Figs. 14 and 15 are shown for the $\{0,1\}$ labeling as they should. 